\title{
Novel functional materials for energy-efficient indoor moisture control
}

\author{
Menghao Qin* \\ Department of Civl Engineering, Technical University of Denmark (DTU), Lyngby, Denmark
}

\begin{abstract}
The regulation of the latent load remains a critical problem for built environment control. Unlike the traditional vapor compression system that features high-energy consumption and environmental-unfriendly processes, desiccants represent an alternative air-conditioning method that takes advantage of the low-grade energy, decreases the energy consumption and even employs use of water vapor. However, for a long time, solid desiccants that can be used for built environment control are very limited. Traditional/conventional desiccants, such as silica gel and zeolite, have relatively low water vapor uptake and high energy demand for desorption, which render them unsuitable for energy-efficient humidity control. In the paper, two types of novel functional materials, i.e. metal-organic frameworks (MOFs) and polymer hydrogels (pHyG) developed at DTU are presented. The hygrothermal and sorption properties of these materials are measured. Both MOFs and pHyG have high water vapor uptake and low regeneration temperature and could be used for energy-efficient indoor moisture control. Some examples of the applications of these new materials developed at DTU are presented. We conclude with prospective directions for next generation solid desiccants to promote energy-efficient moisture control from scientific research to practical application.
\end{abstract} Peer-review under the responsibility of the organizing committee of the ICMB21.

Keywords: Functional materials; MOFs; Hydrogels; Indoor relative humidity; Building energy saving

\section{Introduction/Background}

Latent cooling load accounts for $30 \%$ of the total load of air-conditioning, and its proportion is even higher in many tropical and subtropical climates. Conventional vapor-compression air conditioners normally have a low coefficient of performance (COP) and low energy-efficiency in practice, which is primarily due to the refrigeration dehumidification process. By cooling the air below the dew point, the humidity load is removed by condensation. A great deal of subsequent re-heating is required to increase the air temperature to meet the supply-air requirement for indoor thermal comfort, which wastes a great amount of energy [1]. Considerable efforts have been made to develop alternative dehumidification technologies. Desiccants help the system to independently control both temperature and humidity and thus contribute to the reduction of the energy used [2]. However, for a long time, solid moisture sorbents that can be used for built environment control are very limited. Silica gels and zeolites are the most commonly used solid desiccants, but they are not very efficient for dehumidification owing to their relatively low water vapor uptake, high energy demand for desorption (regeneration temperature higher than $120{ }^{\circ} \mathrm{C}$ ), long cycling time and the fact that a larger part of their water vapor sorption occurs outside the desired comfort relative humidity range. Dehumidification by liquid sorbents uses less electrical energy than refrigeration, but the relevant technology has some inherent defects, e.g. the system is complex and bulky, and has corrosion problems [2]. Therefore, research efforts are going for the development of novel solid desiccants with an ability to improve the energy performance of dehumidification. The building physics group at DTU has been focusing on the development and application of novel functional materials for energy-efficient humidity control, and have prepared a series of novel materials, e.g. metal-organic frameworks (MOFs) based desiccant and polymer hydrogels (pHyG) desiccant etc.

\section{Materials and main features}

Metal-organic frameworks (MOFs) based desiccants MOFs are a new kind of crystalline porous materials constructed by metal-containing inorganic building centers and polyfunctional organic ligands through coordination bonds into three-dimensional interconnected networks. Most MOFs have very high surface areas and large adsorption capacity for gas. Recent studies show that metal-organic frameworks are also promising adsorbents for water vapour [1, 2]. Recently, a new type of MOF based desiccant was prepared at DTU [3]. The material has an S-shape isotherm, large BET surface area and pore volume, and very high water vapor uptake of $1.62 \mathrm{~g} / \mathrm{g}$ at $80 \% \mathrm{RH}$. The regeneration temperature of the new desiccant is around $60{ }^{\circ} \mathrm{C}$.

Polymer hydrogels $(\mathbf{p H y G})$ desiccants The new polymer hydrogel desiccant is prepared by combining hygroscopic materials and hydrophilicity controllable polymers at a molecular level. The water release process can be achieved by a phase separation process, where the polymer in hydrophilic state serving a molecular reservoir is controllably switched to a hydrophobic state, releasing the containing water without any energy-intensive processes such as desorption or dehydration. The maximum water vapor uptake of the polymer hydrogels (pHyG) desiccant can be up to $5.0 \mathrm{~g} / \mathrm{g}$ at $90 \% \mathrm{RH}$.

\footnotetext{
* Corresponding author. Tel.: +45 5036 5768, Email: menqin@byg.dtu.dk, Website: https://www.staff.dtu.dk/menqin/home
} 


\section{ICMB21}

\section{Applications}

The application of the new materials can be generally divided into two categories: passive approaches and active systems. For the passive approaches, the functional materials could be coated on the surface of a wall panel or painted on the wall directly. Fig. 1 shows the sorption isotherms of MOF based desiccant for autonomous regulation of indoor humidity [3]. The material can rapidly adsorb moisture as the indoor relative humidity exceeds $60 \%$, and release moisture as relative humidity drops below $45 \%$.

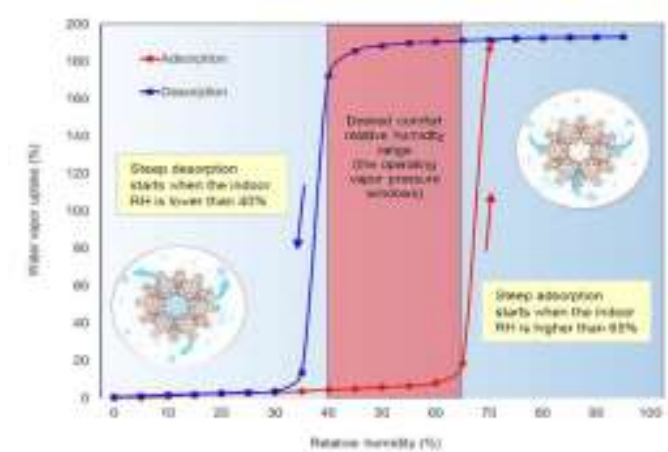

Figure 1. Working principle of the ideal PHCM for autonomous indoor humidity control [3]

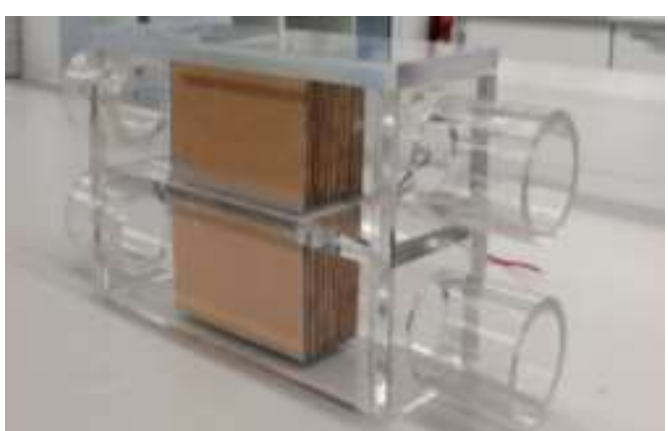

Figure 2. A photo of humidity pump [4]

The new materials could also be integrated with mechanical systems to improve the energy-efficiency of humidity control process. Fig. 2 shows a photo of a device called humidity pump [4], which can transport moisture through the inverse gradient of vapour concentration, i.e., the vapour can be transferred from a relatively low-humidity space to a high-humidity space. Fig. 3 shows the working mechanism of MOF coated heat exchangers for temperature and humidity independent control. The COP of the new system can be up to 7.9. [1]
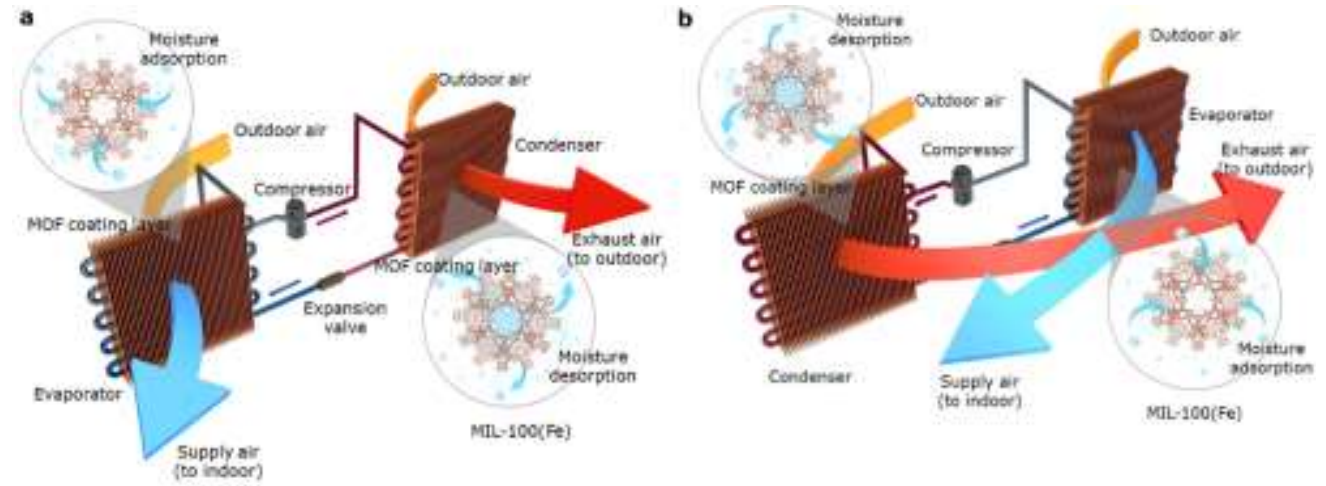

Figure 3. Schematic illustration of the working mechanism of a MOF coated heat pump system [1]

\section{Outlook and opportunities}

Moisture adsorption capacity, the energy demand of desorption, ad/desorption kinetics, and cycling durability are essential features of novel desiccants for moisture control. Superior moisture sorption based on materials with enhanced water affinity, large surface area and high porosity can increase the water uptake to adsorb more moisture from the indoor air. Low regeneration energy demand can be achieved by tailoring the sorption behavior of materials and incorporating functional materials, such as thermal responsive materials to introduce phase separation, which eliminates energy-intensive phase transition process during water collection. In addition, fast water vapor adsorption and desorption are essential for energy-efficient dehumidification within a short period of time.

\section{References}

[1] S. Cui, M. Qin et al. (2018). Metal-Organic Frameworks as advanced moisture sorbents for energy-efficient high temperature cooling, Nature, Scientific Reports, Vol. 8. 15284

[2] K. Zu, M. Qin, S. Cui (2020) Progress and potential of metal-organic frameworks (MOFs) as novel desiccants for built environment control: A review. Renewable \& Sustainable Energy Reviews, Vol. 133. 110246

[3] M. Qin, P. Hou, Z. Wu, J. Huang (2020) Precise humidity control materials for autonomous regulation of indoor moisture, Building and Environment, Vol. 169. 106581

[4] P. Hou, K Zu, M. Qin, S. Cui (2020) A novel metal-organic frameworks based humidity pump for indoor moisture control, Building and Environment, Vol. 187,107396 